\title{
Towards $\bar{B} \rightarrow X_{s} \gamma$ at the NNLO in QCD without interpolation in $m_{c}$
}

\author{
Mikołaj Misiak, ${ }^{a, 1}$ Abdur Rehman ${ }^{b, c}$ and Matthias Steinhauser ${ }^{b}$ \\ ${ }^{a}$ Institute of Theoretical Physics, Faculty of Physics, University of Warsaw, \\ Pasteura 5, 02-093 Warsaw, Poland \\ ${ }^{b}$ Institut für Theoretische Teilchenphysik, Karlsruhe Institute of Technology (KIT), \\ 76128 Karlsruhe, Germany \\ ${ }^{c}$ National Centre for Physics, Quaid-i-Azam University Campus, \\ 45320 Islamabad, Pakistan \\ E-mail: Mikolaj.Misiak@fuw.edu.pl, Abdur.Rehman@ncp.edu.pk, \\ Matthias.Steinhauser@kit.edu
}

ABSTRACT: Strengthening constraints on new physics from the $\bar{B} \rightarrow X_{s} \gamma$ branching ratio requires improving accuracy in the measurements and the Standard Model predictions. To match the expected Belle-II accuracy, Next-to-Next-to-Leading Order (NNLO) QCD corrections must be calculated without the so-far employed interpolation in the charmquark mass $m_{c}$. In the process of evaluating such corrections at the physical value of $m_{c}$, we have finalized the part coming from diagrams with closed fermion loops on the gluon lines that contribute to the interference of the current-current and photonic dipole operators. We confirm several published results for corrections of this type, and supplement them with a previously uncalculated piece. Taking into account the recently improved estimates of non-perturbative contributions, we find $\mathcal{B}_{s \gamma}=(3.40 \pm 0.17) \times 10^{-4}$ and $R_{\gamma} \equiv \mathcal{B}_{(s+d) \gamma} / \mathcal{B}_{c \ell \bar{\nu}}=$ $(3.35 \pm 0.16) \times 10^{-3}$ for $E_{\gamma}>1.6 \mathrm{GeV}$ in the decaying meson rest frame.

KEYwords: Heavy Quark Physics, Perturbative QCD

ArXiv EPRINT: 2002.01548

\footnotetext{
${ }^{1}$ Corresponding author.
} 


\section{Contents}

1 Introduction 1

2 The NNLO contribution to $\hat{G}_{27} \quad 4$

3 Updated SM predictions for $\mathcal{B}_{s \gamma}$ and $\boldsymbol{R}_{\gamma} \quad 7$

$\begin{array}{lll}4 & \text { Summary } & 11\end{array}$

A Large- $z$ expansions and $\hat{G}_{47}^{(1)}$ with charm loops 12

\section{Introduction}

Flavour Changing Neutral Current (FCNC) processes receive the leading Standard Model (SM) contributions from one-loop diagrams only, often with additional suppression factors originating from the Glashow-Iliopoulos-Maiani (GIM) mechanism [1]. It makes them sensitive to possible existence of new weakly-interacting particles with masses ranging up to $\mathcal{O}(100 \mathrm{TeV})$. Significant deviations from the SM predictions are observed in the GIMunsuppressed FCNC processes mediated by the $b \rightarrow s \mu^{+} \mu^{-}$transition (see, e.g., the recent summary in ref. [2]). On the other hand, no deviations are seen in the closely related $b \rightarrow s \gamma$ transition, despite higher accuracy of both the measurements and the SM predictions in its case.

The physical observable giving the strongest constraints on the $b \rightarrow s \gamma$ amplitude is the inclusive $\mathcal{B}_{s \gamma}$ branching ratio, i.e. the CP- and isospin- averaged branching ratio of $\bar{B} \rightarrow X_{s} \gamma$ and $B \rightarrow X_{\bar{s}} \gamma$ decays, with $\bar{B}$ and $B$ denoting $\left(\bar{B}^{0}\right.$ or $\left.B^{-}\right)$and $\left(B^{0}\right.$ or $\left.B^{+}\right)$, respectively. The states $X_{s}$ and $X_{\bar{s}}$ are assumed to contain no charmed hadrons. $\mathcal{B}_{s \gamma}$ is being measured [3-8] with $E_{\gamma}>E_{0}$ for $E_{0} \in[1.7,2.0] \mathrm{GeV}$, and then extrapolated to the conventionally chosen value of $E_{0}=1.6 \mathrm{GeV}$ to compare with the theoretical predictions (that would be less accurate at higher $E_{0}$ ). The current experimental world average for $\mathcal{B}_{s \gamma}$ at $E_{0}=1.6 \mathrm{GeV}$ reads $(3.32 \pm 0.15) \times 10^{-4}$ [9], which corresponds to an uncertainty of around $\pm 4.5 \%$. With the full Belle-II dataset, the world average uncertainty at the level of $\pm 2.6 \%$ is expected $[10,11]$. Achieving a similar accuracy in the SM predictions is essential for improving the power of $\mathcal{B}_{s \gamma}$ as a constraint on Beyond-SM (BSM) theories. It is the goal of the calculations we describe in what follows.

The SM prediction for $\mathcal{B}_{s \gamma}$ (see refs. $[12,13]$ ), is based on the formula

$$
\mathcal{B}\left(\bar{B} \rightarrow X_{s} \gamma\right)_{E_{\gamma}>E_{0}}=\mathcal{B}\left(\bar{B} \rightarrow X_{c} \ell \bar{\nu}\right)\left|\frac{V_{t s}^{*} V_{t b}}{V_{c b}}\right|^{2} \frac{6 \alpha_{\mathrm{e} m}}{\pi C}\left[P\left(E_{0}\right)+N\left(E_{0}\right)\right],
$$


where $\alpha_{\mathrm{e} m}=\alpha_{\mathrm{e} m}^{\mathrm{on} \text { shell }}$, while the so-called semileptonic phase-space factor $C$ is given by

$$
C=\left|\frac{V_{u b}}{V_{c b}}\right|^{2} \frac{\Gamma\left[\bar{B} \rightarrow X_{c} e \bar{\nu}\right]}{\Gamma\left[\bar{B} \rightarrow X_{u} e \bar{\nu}\right]}
$$

Its numerical value is determined [14] using the Heavy Quark Effective Theory (HQET) methods from measurements of the $\bar{B} \rightarrow X_{c} \ell \bar{\nu}$ decay spectra. The quantity $P\left(E_{0}\right)$ is defined through the following ratio of perturbative inclusive decay rates of the $b$ quark:

$$
\frac{\Gamma\left[b \rightarrow X_{s}^{p} \gamma\right]_{E_{\gamma}>E_{0}}}{\left|V_{c b} / V_{u b}\right|^{2} \Gamma\left[b \rightarrow X_{u}^{p} e \bar{\nu}\right]}=\left|\frac{V_{t s}^{*} V_{t b}}{V_{c b}}\right|^{2} \frac{6 \alpha_{\mathrm{e} m}}{\pi} P\left(E_{0}\right),
$$

with $X_{s}^{p}$ and $X_{u}^{p}$ denoting all the possible charmless partonic final states in the respective decays $\left(X_{s}^{p}=s, s g, s q \bar{q}, \ldots\right)$. The non-perturbative contribution from $N\left(E_{0}\right)$ in eq. (1.1) is estimated $^{1}$ at the level of around $4 \%$ of $\mathcal{B}_{s \gamma}$. To achieve $\mathcal{O}(3 \%)$ precision in $P\left(E_{0}\right)$, evaluation of the Next-to-Next-to Leading (NNLO) QCD corrections to this quantity is necessary.

Perturbative calculations of $P\left(E_{0}\right)$ are most conveniently performed in the framework of an effective theory obtained from the SM via decoupling of the $W$ boson and all the heavier particles. The relevant weak interactions are then given by the following Lagrangian density $^{2}$

$$
\mathcal{L}_{\text {weak }}=\frac{4 G_{F}}{\sqrt{2}} V_{t s}^{\star} V_{t b} \sum_{i=1}^{8} C_{i}\left(\mu_{b}\right) Q_{i} .
$$

Evaluation of the Wilson coefficients $C_{i}$ to the NNLO accuracy $\left(\mathcal{O}\left(\alpha_{\mathrm{s}}^{2}\right)\right)$ at the renormalization scale $\mu_{b} \sim m_{b}$ required computing electroweak-scale matching up to three loops [15], and QCD anomalous dimensions up to four loops [16]. Since $C_{i}$ in the SM have no imaginary parts, one can write the perturbative decay rate as

$$
\Gamma\left(b \rightarrow X_{s}^{p} \gamma\right)=\frac{G_{F}^{2} m_{b, \text { pole }}^{5} \alpha_{\mathrm{e} m}}{32 \pi^{4}}\left|V_{t s}^{*} V_{t b}\right|^{2} \sum_{i, j=1}^{8} C_{i}\left(\mu_{b}\right) C_{j}\left(\mu_{b}\right) \hat{G}_{i j}, \quad\left(\hat{G}_{i j}=\hat{G}_{j i}\right),
$$

where $\hat{G}_{i j}$ come from interferences of amplitudes with insertions of the operators $Q_{i}$ and $Q_{j}$. The dominant NNLO effects come from $\hat{G}_{17}, \hat{G}_{27}$ and $\hat{G}_{77}$ that originate from the operators

$$
Q_{1}=\left(\bar{s}_{L} \gamma_{\mu} T^{a} c_{L}\right)\left(\bar{c}_{L} \gamma^{\mu} T^{a} b_{L}\right), \quad Q_{2}=\left(\bar{s}_{L} \gamma_{\mu} c_{L}\right)\left(\bar{c}_{L} \gamma^{\mu} b_{L}\right), \quad Q_{7}=\frac{e m_{b}}{16 \pi^{2}}\left(\bar{s}_{L} \sigma^{\mu \nu} b_{R}\right) F_{\mu \nu}
$$

Whereas $\hat{G}_{77}$ has been known up to $\mathcal{O}\left(\alpha_{\mathrm{s}}^{2}\right)$ since a long time [17-21], no complete NNLO calculation of $\hat{G}_{17}$ and $\hat{G}_{27}$ at the physical value of the charm quark mass $m_{c}$ has been finalized to date. Instead, calculations of these quantities at $m_{c} \gg m_{b}[22,23]$ and $m_{c}=0$ [13] gave a basis for estimating their physical values using interpolation [13]. The related uncertainty in $\mathcal{B}_{s \gamma}$ (due to the $m_{c}$-interpolation only) has been estimated at the level of $\pm 3 \%$,

\footnotetext{
${ }^{1}$ See section 3 for details on the current uncertainty budget.

${ }^{2}$ For simplicity, we refrain here from displaying those terms in $\mathcal{L}_{\text {weak }}$ that matter for subleading electroweak or CKM-suppressed effects only. Such effects have been included in the numerical analysis of refs. [12, 13].
} 

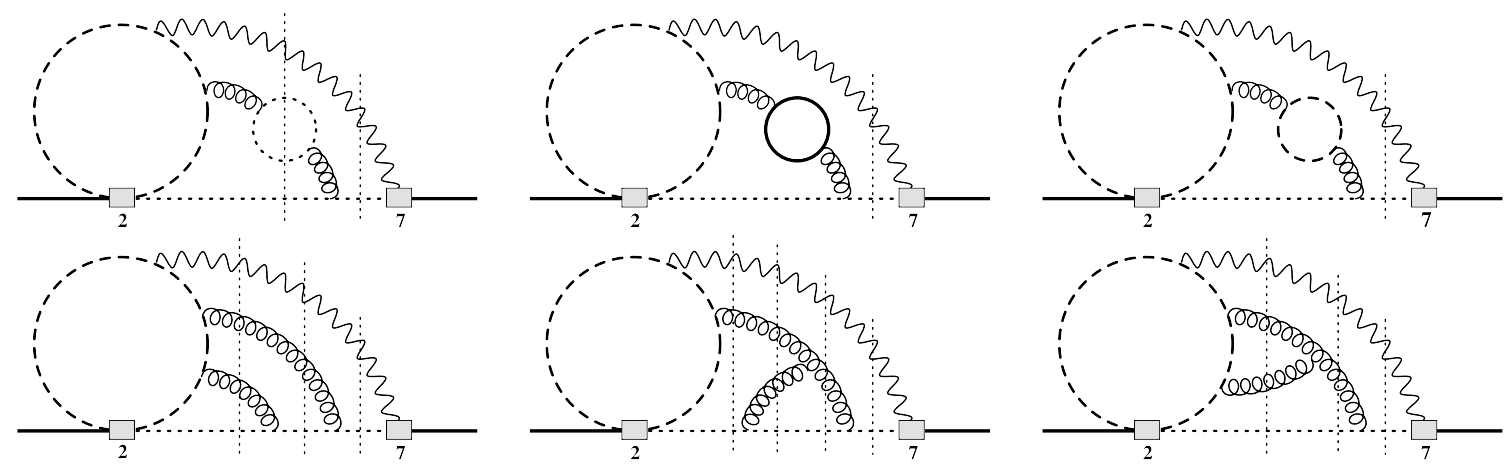

Figure 1. Sample Feynman diagrams contributing to $\hat{G}_{27}^{(2)}$ at $\mathcal{O}\left(\alpha_{\mathrm{s}}^{2}\right)$. The vertical dotted lines indicate possible unitarity cuts. The dotted, dashed and solid propagators correspond to quarks with masses $0, m_{c}$ and $m_{b}$, respectively.

which places it among the dominant contributions to the overall theoretical uncertainty (see section 3).

To calculate the interferences $\hat{G}_{i j}$ at the physical value of $m_{c}$, it is convenient to express them in terms of propagator diagrams with unitarity cuts. Examples of such fourloop diagrams contributing to $\hat{G}_{27}$ at $\mathcal{O}\left(\alpha_{\mathrm{s}}^{2}\right)$ are shown in figure 1 , with the light quarks $(u, d, s)$ treated as massless. Similar diagrams for $\hat{G}_{17}$ differ from the $\hat{G}_{27}$ ones by simple colour factors only. For definiteness, we shall focus on $\hat{G}_{27}$ in what follows.

By analogy to what has been done in the $\hat{G}_{77}$ case [17-21], evaluation of $\mathcal{O}\left(\alpha_{\mathrm{s}}^{2}\right)$ contributions to $\hat{G}_{27}$ is performed in two steps. First, no restriction on the photon energy $E_{\gamma}$ is assumed. Next, one performs the calculation for $E_{\gamma}<E_{0}$, which requires considering diagrams with three- and four-body cuts only. The desired result $\hat{G}_{27}^{E_{\gamma}>E_{0}}=\hat{G}_{27}^{\text {any } E_{\gamma}}-\hat{G}_{27}^{E_{\gamma}<E_{0}}$ is then obtained without necessity of determining the differential photon spectrum close to the endpoint $E_{\gamma}^{\max }=\frac{1}{2} m_{b}$.

In the present paper, we describe our calculation of $\hat{G}_{27}^{(2)}$ in

$$
\hat{G}_{27}=\frac{\alpha_{\mathrm{s}}}{4 \pi} \hat{G}_{27}^{(1)}+\left(\frac{\alpha_{\mathrm{s}}}{4 \pi}\right)^{2} \hat{G}_{27}^{(2)}+\mathcal{O}\left(\alpha_{\mathrm{s}}^{3}\right)
$$

at the physical value of $m_{c}$, and with no restriction on $E_{\gamma}$. Final results are presented for contributions originating from diagrams with closed fermion loops on the gluon lines, like those in the first row of figure 1. They undergo separate renormalization and are gauge invariant on their own, so they serve as a useful test case for our calculation of the complete $\hat{G}_{27}^{(2)}$. Most of such contributions have already been determined in the past [24-27] and implemented in the phenomenological analysis $[12,13]$. We confirm the published results, and supplement them with a previously uncalculated piece. Some of the previous results have been obtained by a single group only, which makes our verification relevant.

The article is organized as follows. In the next section, our algorithm for evaluation of the complete $\hat{G}_{27}^{(2)}$ is sketched, and the current status of the calculation is summarized. Next, we focus on the closed fermionic loop contributions, displaying our numerical results and comparing them with the literature wherever possible. In section 3, the SM prediction for the branching ratio is updated, taking into account the recently improved estimates of 
non-perturbative effects [28]. We conclude in section 4. In the appendix, large- $z$ expansions of our final results are presented, and one of the counterterm contributions is discussed.

\section{The NNLO contribution to $\hat{G}_{27}$}

The quantity $\hat{G}_{27}^{(2)}$ is given by a few hundreds of four-loop propagator diagrams with unitarity cuts, as those presented in figure 1. We generate them using QGRAF [29] and/or FeynArts [30, 31]. After performing the Dirac algebra with the help of FORM [32], we express the full $\hat{G}_{27}^{(2)}$ in terms of several hundred thousands scalar integrals grouped in $\mathcal{O}(500)$ families. ${ }^{3}$ Next, the Integration By Parts (IBP) identities [33-35] for each family are generated and applied using KIRA [36, 37], as well as FIRE [38, 39] and LiteRed [40, 41]. In effect, $\hat{G}_{27}^{(2)}$ becomes a linear combination of Master Integrals (MIs). The IBP reduction is the most computer-power demanding part of the calculation, with $\mathcal{O}(1 \mathrm{~TB}) \mathrm{RAM}$ nodes and weeks of CPU time needed for the most complicated families.

After setting the renormalization scale squared to $\mu_{b}^{2}=e^{\gamma} m_{b}^{2} /(4 \pi)$ (with $\gamma$ being the Euler-Mascheroni constant), the MIs are multiplied by appropriate powers of $m_{b}$, to make them dimensionless. They depend on two parameters only: the dimensional regularization parameter $\epsilon$, and the quark mass ratio $z=m_{c}^{2} / m_{b}^{2}$. In each family separately, the MIs $M_{k}(z, \epsilon)$ satisfy the Differential Equations (DEs)

$$
\frac{d}{d z} M_{k}(z, \epsilon)=\sum_{l} R_{k l}(z, \epsilon) M_{l}(z, \epsilon)
$$

where the rational functions $R_{k l}(z, \epsilon)$ on the r.h.s. are determined [42-44] from the IBP, too. ${ }^{4}$ Similar equations are explicitly displayed in eq. (3.6) of ref. [45] where ultraviolet counterterm contributions to $\hat{G}_{27}^{(2)}$ have been determined.

We solve the DEs using the same method as in refs. [26, 45, 46]. The MIs are expanded in $\epsilon$ to appropriate powers, with the expansion coefficients being functions of $z$ only. Boundary conditions for these functions at large $z$ are found using asymptotic expansions [47]. Next, the variable $z$ is treated as complex, and the DEs are numerically solved along half-ellipses in the $z$-plane, to bypass singularities on the real axis.

In practice, the codes $\mathrm{q} 2 \mathrm{e}$ and $\exp [48,49]$ are used to determine the asymptotic expansions at large $z$. Coefficients at subsequent powers of $1 / z$ are given in terms of one-, two- and three-loop single-scale integrals, either massive tadpoles or propagator-type ones with unitarity cuts (see figure 2). Only at the level of the latter integrals, we perform cross-family identification, which gives us $\mathcal{O}(50)$ essentially different and non-vanishing integrals. They are evaluated [50] using various techniques, in particular the Mellin-Barnes one. Once the large- $z$ expansions are found, numerical solutions of the DEs starting from the boundary at $z=20$ are worked out using the code ZVODE [51] upgraded to quadrupoledouble precision with the help of the QD [52] computation package. Half-ellipses of various sizes are considered to test the numerical stability.

\footnotetext{
${ }^{3}$ Integrals in a family differ only by indices, i.e. the powers to which the propagators and/or irreducible numerators are being raised.

${ }^{4}$ Getting a closed system of such DEs usually requires including several new MIs w.r.t. those entering the expression for $\hat{G}_{27}^{(2)}$.
} 


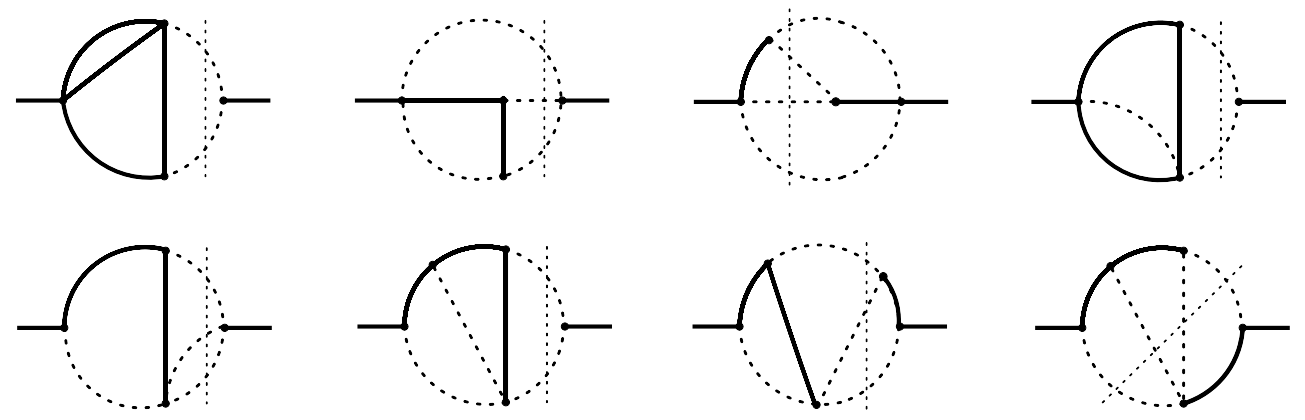

Figure 2. Sample three-loop propagator-type integrals that parameterize large- $z$ expansions of the MIs. Massless and massive internal propagators are denoted by dotted and solid lines, respectively. The thin dotted lines indicate the unitarity cuts.
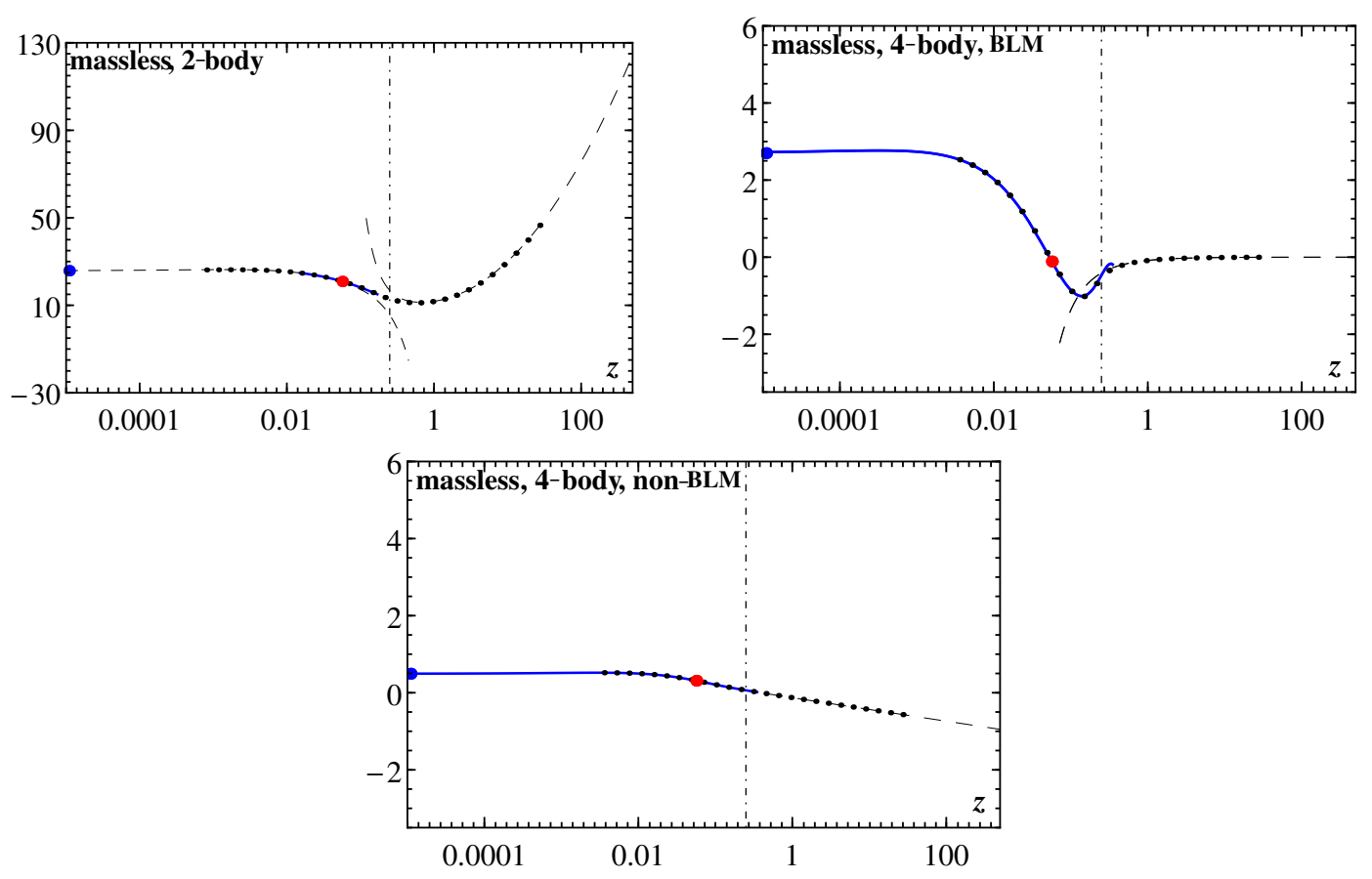

Figure 3. Contributions to $\hat{G}_{27}^{(2)}$ from diagrams with closed loops of massless fermions - see the text. They have already been multiplied by $n_{l}=3$, i.e. the number of flavours we treat as massless.

At present, our IBP reduction for the full $\hat{G}_{27}^{(2)}$ is (almost) completed, and the evaluation of the boundary conditions is being finalized [50]. However, for the diagrams with closed fermionic loops (as the ones in the first row of figure 1), the DEs are already solved, and we are ready to present the final results. They are plotted in figures 3 and 4 as functions of $z$.

The displayed results correspond to various contributions to $\hat{G}_{27}^{(2)}$ renormalized in the $\overline{\mathrm{MS}}$ scheme with $\mu_{b}^{2}=m_{b}^{2}$ (or, equivalently, in the MS scheme with $\mu_{b}^{2}=e^{\gamma} m_{b}^{2} /(4 \pi)$ ). The renormalization has been performed with the help of the counterterm contributions evaluated ${ }^{5}$ in refs. $[45,46]$. In all the plots, the black dots correspond to numerical solutions that we have obtained using the DEs. Dots corresponding to the physical value of $z$ are

\footnotetext{
${ }^{5}$ In the charm loop case (the right plot in figure 4), we had to rely on our so-far unpublished results for the UV counterterms - see the appendix.
} 

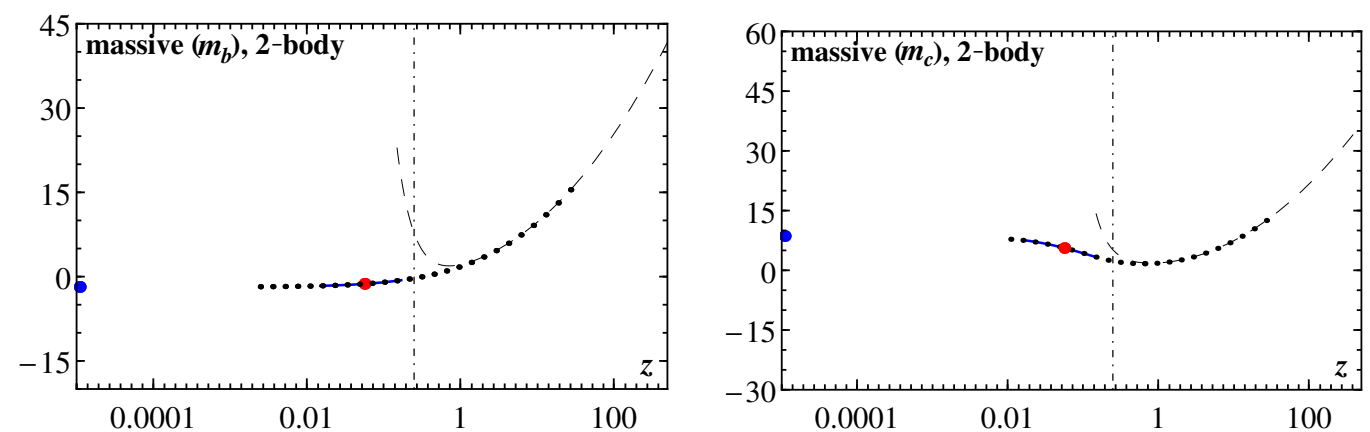

Figure 4. Contributions to $\hat{G}_{27}^{(2)}$ from diagrams with closed loops of massive fermions - see the text.
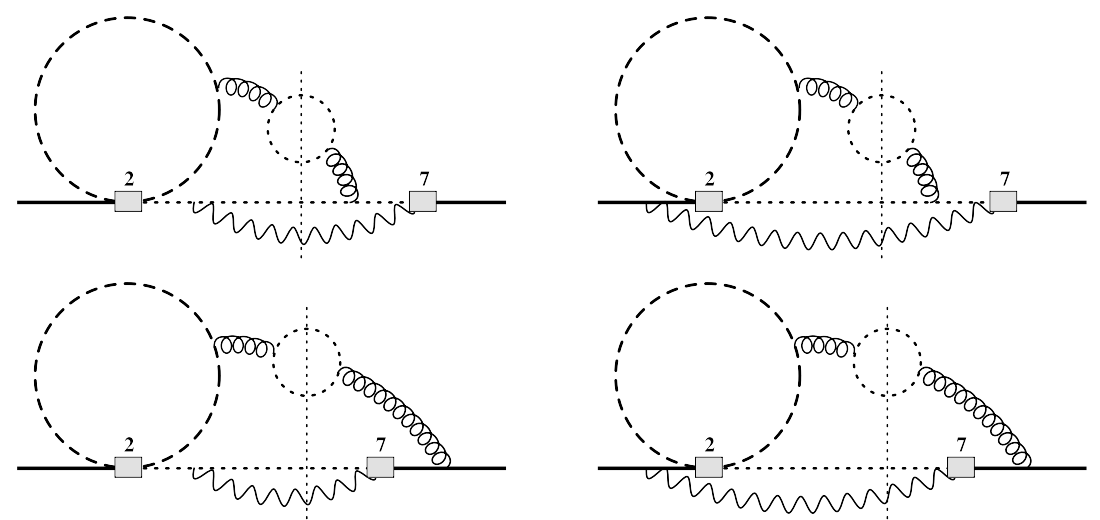

Figure 5. Diagrams corresponding to the last (lower) plot in figure 3.

bigger and highlighted in red. Blue dots of similar size on the left boundaries of each plot indicate the $z \rightarrow 0$ limits for each contribution, known from the calculation in ref. [13]. Thin dashed curves continuing to large values of $z$ describe our large- $z$ expansions evaluated up to $\mathcal{O}\left(1 / z^{2}\right)$ (see the appendix). The dash-dotted vertical lines indicate the $c \bar{c}$ production threshold at $z=1 / 4$, in the vicinity of which neither the large- $z$ nor the small- $z$ expansions are expected to converge well.

In figure 3, three distinct contributions from diagrams with closed massless fermion loops are presented. The first (upper left) plot corresponds to diagrams with two-body cuts. The thin dashed line in the small- $z$ region shows the analytic expansion in powers of $z$ evaluated in ref. [25]. It is the only case for which such an expansion is known. The solid blue curve shows the numerical fit corresponding to eq. (3.2) of ref. [26] where a numerical method (identical to ours) has been used.

The second (upper right) plot of figure 3 shows all the four-body-cut contributions except the diagrams displayed in figure 5 . The latter diagrams have been skipped ${ }^{6}$ in evaluating the photon spectrum in the Brodsky-Lepage-Mackenzie (BLM) [53] approximation

\footnotetext{
${ }^{6}$ Arguments in favour of not including them in the BLM approach can be found below eq. (12) of ref. [24]. They are correlated via renormalization group with tree-level $b \rightarrow s q \bar{q} \gamma$ matrix elements of the penguin four-quark operators.
} 
by the authors of refs. [24, 27]. The solid blue curve is based on the numerical fit from eq. (3.6) of ref. [13] that corresponds to no restriction on $E_{\gamma}$, and has been obtained as a by-product of the calculation in ref. [27].

The third (bottom) plot in figure 3 corresponds to the very diagrams from figure 5 . In this case, no numerical result valid for arbitrary $m_{c}$ has existed prior to our present calculation. For $z<\frac{1}{4}$, we can describe our findings by the following fit:

$$
\Delta_{m=0}^{4-\mathrm{b} \mathrm{BEM}} \hat{G}_{27}^{(2)}=3\left[0.164+0.13 z^{\frac{1}{2}}-21.51 z+68.10 z^{\frac{3}{2}}-46.12 z^{2}+\left(-3.23 z+18.23 z^{2}\right) \ln z\right] .
$$

It is shown as a solid blue curve in the considered plot. A quick look at figure 5 is sufficient to realize that $\Delta_{m=0}^{4-b \text { BEस }} \hat{G}_{17}^{(2)}=-\frac{1}{6} \Delta_{m=0}^{4 \text {-b BEस }} \hat{G}_{27}^{(2)}$, due to the identity $T^{a} T^{b} T^{a}=-\frac{1}{6} T^{b}$ for the $\mathrm{SU}(3)_{c}$ generators. The same relative colour factor is valid for all the plots in figures 3 and 4 .

Figure 4 shows contributions to $\hat{G}_{27}^{(2)}$ from diagrams with closed loops of quarks with masses $m_{b}$ (left) and $m_{c}$ (right). Only the two-body cuts are included. The solid blue lines correspond to the numerical fits from eqs. (3.3) and (3.4) of ref. [26]. In these cases, no four-body cuts are allowed, as the state $X_{s}^{p}$ in eq. (1.5) is assumed to contain no charm quarks. We do not consider three-body cuts here, as their effect can be included by multiplying the well-known three-body contribution to $\hat{G}_{27}^{(1)}$ by finite coefficients originating from $^{7} Z_{G}^{\mathrm{OS}} Z_{g}^{2}-1$. The corresponding term in eq. (3.8) of ref. [13] comes at the end of the first line of the expression for $K_{27}^{(2)}$.

As evident from the plots, our results are in perfect agreement with all the previously available expansions and fits. It is particularly important in the massive case (figure 4) where our verification comes as the first one from an independent group. Let us note that the contribution displayed in the right plot of figure 4 affects $\mathcal{B}_{s \gamma}$ by around $-2.1 \%$, which should be compared to the current $( \pm 4.5 \%)$ and expected future $( \pm 2.6 \%)$ experimental accuracies mentioned in section 1 . The massless results from the upper two plots of figure 3 have already been cross-checked before.

As far as the new contribution (the third plot in figure 3) is concerned, it has so far been included in the interpolated part of the NNLO correction, and resulted in a tiny effect, around one per-mille of the decay rate only. Now we remove it from the interpolated part and replace by the fit in eq. (2.2). It turns out that the interpolation estimate was correct within $\sim 10 \%$ of the considered contribution, so the effect remains tiny.

\section{Updated SM predictions for $\mathcal{B}_{s \gamma}$ and $\boldsymbol{R}_{\gamma}$}

In the present section, we work out updated SM predictions for $\mathcal{B}_{s \gamma}$, as well as for the ratio $R_{\gamma} \equiv \mathcal{B}_{(s+d) \gamma} / \mathcal{B}_{c \ell \bar{\nu}}$, where $\mathcal{B}_{c \ell \bar{\nu}}$ is the CP- and isospin-averaged branching ratio of the inclusive semileptonic decay. Our main motivation for performing an update right now is not due to the NNLO corrections evaluated in the previous section. The new contribution is tiny, while the sizeable ones (that we have confirmed) were already included in the

\footnotetext{
${ }^{7} Z_{G}^{\text {OS }}$ stands for the on-shell renormalization constant of the gluon wave-function, while $Z_{g}$ renormalizes the QCD gauge coupling in the MS scheme.
} 
phenomenological analysis of ref. [13]. However, there has been an important progress in estimating non-perturbative effects (see below). An update of the SM prediction should thus be performed right now, even though the $m_{c}$-interpolation uncertainty remains essentially unchanged.

The first improvement in estimating the non-perturbative effects becomes possible thanks to the new Belle measurement of the isospin asymmetry

$$
\Delta_{0-} \equiv \frac{\Gamma\left[\bar{B}^{0} \rightarrow X_{s} \gamma\right]-\Gamma\left[B^{-} \rightarrow X_{s} \gamma\right]}{\Gamma\left[\bar{B}^{0} \rightarrow X_{s} \gamma\right]+\Gamma\left[B^{-} \rightarrow X_{s} \gamma\right]}=(-0.48 \pm 1.49 \pm 0.97 \pm 1.15) \%[54] .
$$

In the SM, the dominant contribution to this asymmetry arises from a process where no hard photon but rather a hard ${ }^{8}$ gluon is emitted in the $b$-quark decay [55]. Next, the gluon scatters on the valence quark, which results in emission of a hard photon. Instead of the valence quark, also a sea quark $(u, d$ or $s)$ can participate in such a Compton-like scattering. Taking this fact into account, one can write the decay rates as

$$
\begin{aligned}
& \Gamma\left[B^{-} \rightarrow X_{s} \gamma\right] \simeq A+B Q_{u}+C Q_{d}+D Q_{s}, \\
& \Gamma\left[\bar{B}^{0} \rightarrow X_{s} \gamma\right] \simeq A+B Q_{d}+C Q_{u}+D Q_{s}
\end{aligned}
$$

where $Q_{u, d, s}$ denote electric charges of the quarks participating in the Compton-like scattering, while the quantities $A, \ldots, D$ are given by interferences of various quantum amplitudes whose explicit form is inessential here. Since the considered effect gives only a small correction to the decay rate $(B, C, D \ll A)$, quadratic terms in $Q_{u, d, s}$ have been neglected above. We have also neglected isospin violation in the quark masses $\left(m_{u} \neq m_{d}\right)$ and in the electromagnetic corrections to the $\bar{B}$-meson wave functions (suppressed by extra powers of $\alpha_{e m}$ ).

The leading term $A$ contains the dominant contribution originating from the operator $Q_{7}$. The corrections $B, C, D$ are suppressed w.r.t. $A$ both by $g_{s}^{2}$ (as the gluon is hard) and by $\Lambda / m_{b}$, with $\Lambda \sim \Lambda_{\mathrm{QCD}}$. The latter suppression can be intuitively understood by realizing that the gluon scatters on remnants of the $\bar{B}$ meson, i.e. on a diluted target whose size scales like $1 / \Lambda$. Such a suppression is confirmed in refs. $[55,56]$ where the Soft-Collinear Effective Theory (SCET) has been applied to analyze non-perturbative corrections to $\mathcal{B}_{s \gamma}$.

From eq. (3.2), one easily obtains the isospin-averaged decay rate

$$
\Gamma \simeq A+\frac{1}{2}(B+C)\left(Q_{u}+Q_{d}\right)+D Q_{s} \equiv A+\delta \Gamma_{c},
$$

and the isospin asymmetry

$$
\Delta_{0-} \simeq \frac{C-B}{2 \Gamma}\left(Q_{u}-Q_{d}\right) .
$$

It follows that the relative correction to the isospin-averaged decay rate that arises due to the considered effect reads

$$
\frac{\delta \Gamma_{c}}{\Gamma} \simeq \frac{(B+C)\left(Q_{u}+Q_{d}\right)+2 D Q_{s}}{(C-B)\left(Q_{u}-Q_{d}\right)} \Delta_{0-}=\frac{Q_{u}+Q_{d}}{Q_{d}-Q_{u}}\left[1+2 \frac{D-C}{C-B}\right] \Delta_{0-},
$$

where, in the last step, $Q_{s}=-Q_{u}-Q_{d}$ has been used. The second term in the square bracket vanishes in the $\mathrm{SU}(3)_{F}$ limit, i.e. when the three lightest quarks are treated as

\footnotetext{
${ }^{8}$ With momentum of order $m_{b}$ but possibly much smaller virtuality.
} 
mass-degenerate. In this limit, as observed in ref. [57], $\delta \Gamma_{c} / \Gamma$ and $\Delta_{0-}$ are related to each other in a simple manner that is free from non-perturbative uncertainties. The authors of ref. [56] suggested $\pm 30 \%$ as an uncertainty estimate stemming from the $\mathrm{SU}(3)_{F}$-violating effect in eq. (3.5). Following this suggestion, we find

$$
\frac{\delta \Gamma_{c}}{\Gamma}=-\frac{1}{3}(1 \pm 0.3) \Delta_{0-}=(0.16 \pm 0.74) \%,
$$

where the experimental errors from eq. (3.1) were combined in quadrature, giving $\Delta_{0-}=(-0.48 \pm 2.12) \%$; next, the multiplicative factor was taken into account as follows [58]:

$$
(1 \pm 0.3)(-0.48 \pm 2.12) \%=\left(-0.48 \pm \sqrt{2.12^{2}+(0.3 \cdot 0.48)^{2}+(0.3 \cdot 2.12)^{2}}\right) \% .
$$

In the above considerations, we have treated the measured $\Delta_{0-}$ in eq. (3.1) as already extrapolated from the experimental cutoff of $E_{0}=1.9 \mathrm{GeV}$ down to our default $E_{0}=1.6 \mathrm{GeV}$, even though no such extrapolation has actually been done in ref. [54], i.e. eq. (3.1) corresponds to $E_{0}=1.9 \mathrm{GeV}$. A devoted analysis would be necessary to estimate the extrapolation effects in this case. However, we expect such effects to be negligible w.r.t. the experimental uncertainties in eq. (3.1).

If the uncertainty on the r.h.s. of eq. (3.6) is treated as $1 \sigma$ of a Gaussian distribution, then the $95 \%$ C.L. range is $[-1.3,+1.6] \%$. The corresponding ${ }^{9}$ range $[-1.4,+2.0] \%$ in section 3.5 of ref. [28] is somewhat wider due to a different method of combining uncertainties and using the PDG $[59,60]$ central value of $-0.6 \%$ for $\Delta_{0-}$. When determining our SM predictions below, we calculate $\mathcal{B}_{s \gamma}$ without including the photon emission from the valence/sea quarks and, in the final step, we multiply by $\left(1+\frac{\delta \Gamma_{c}}{\Gamma}\right)$, employing the number from the r.h.s. of eq. (3.6).

Another important non-perturbative correction to be considered arises in the interference of $Q_{1,2}$ and $Q_{7}$. Its presence in the inclusive $\bar{B} \rightarrow X_{s} \gamma$ rate was first pointed out in ref. [61]. It amounts to around $+3 \%$ of $\mathcal{B}_{s \gamma}$, as established in ref. [62] at the leading order of an expansion in powers of $m_{b} \Lambda / m_{c}^{2}$. The corresponding leading contribution to $N\left(E_{0}\right)$ in eq. (1.1) reads

$$
\delta N_{V}=-\frac{\mu_{G}^{2}}{27 m_{c}^{2}} C_{7}\left(\mu_{b}\right)\left(C_{2}\left(\mu_{b}\right)-\frac{1}{6} C_{1}\left(\mu_{b}\right)\right),
$$

where $\mu_{G}^{2} \simeq 0.3 \mathrm{GeV}^{2}$ is one of the HQET parameters that matter in the determination of $C$ in eq. (1.2). Since $m_{b} \Lambda / m_{c}^{2}$ is not a small parameter, the authors of ref. [56] argued that no expansion in its powers can be used at all. Instead, they estimated the considered correction in the framework of SCET, where essential constraints on models of the relevant soft function came from moments of the semileptonic $\bar{B} \rightarrow X_{c} \ell \bar{\nu}$ decay spectra. A recent update of these estimates in ref. [28] implies that $\delta N_{V}$ (3.8) needs to be multiplied by

$$
\kappa_{V}=1-\frac{27 m_{c}^{2} \Lambda_{17}}{m_{b} \mu_{G}^{2}}=1.2 \pm 0.3 .
$$

The final numerical value above has been derived by us from ranges for $\Lambda_{17}$ given in ref. [28], assuming that these ranges can be interpreted as $1 \sigma$ ones. The remaining parameters on

\footnotetext{
${ }^{9}$ Our $\delta \Gamma_{c} / \Gamma$ and their $\mathcal{F}_{78}^{\exp }$ are estimated in a similar way.
} 
which $\kappa_{V}$ depends were set to the values corresponding to the widest range for $\Lambda_{17}$ in ref. [28].

Since the expression for $\delta N_{V}$ (3.8) is calculated at the leading order in QCD only, the renormalization scheme for $m_{c}^{2}$ in the denominator is unspecified. We assume that the corresponding uncertainty is included in the overall $\pm 3 \%$ higher-order one that is being retained the same as in ref. [13]. As the total effect from $\delta N_{V}$ amounts to around $3 \%$ in $\mathcal{B}_{s \gamma}$, uncertainties due to scheme-dependence of $m_{c}$ in $\delta N_{V}$ can safely be treated this way. In our numerical calculations, the quark masses and HQET parameters are included with a full correlation matrix (see appendix D of ref. [13]), except for the very $m_{c}$ in $\delta N_{V}$ that is now fixed to $1.17 \mathrm{GeV}$. The parameter $\kappa_{V}(3.9)$ will be treated as uncorrelated.

Apart from the two effects we have discussed above, the authors of ref. [56] identified a third source of uncertain contributions to $N\left(E_{0}\right)$ that arise at the order $\mathcal{O}\left(\Lambda / m_{b}\right)$. They come proportional to $\left|C_{8}\left(\mu_{b}\right)\right|^{2}$, where $C_{8}$ is the Wilson coefficient of the gluonic dipole operator

$$
Q_{8}=\frac{g_{s} m_{b}}{16 \pi^{2}}\left(\bar{s}_{L} \sigma^{\mu \nu} T^{a} b_{R}\right) G_{\mu \nu}^{a} .
$$

Previous estimates of these corrections in refs. [63, 64] focused on large collinear logarithms $\ln \frac{m_{b}}{m_{s}}$ that are present in the corresponding contributions to $P\left(E_{0}\right)$. In ref. [13], such logarithms were varied in the range $[\ln 10, \ln 50] \simeq\left[\ln \frac{m_{B}}{m_{K}}, \ln \frac{m_{B}}{m_{\pi}}\right]$, which served as a crude estimate of the very uncertain but otherwise small contributions to $\mathcal{B}_{s \gamma}$ where light hadron masses are the physical collinear regulators. However, according to ref. [56], possible non-perturbative effects that come multiplied by $\left|C_{8}\left(\mu_{b}\right)\right|^{2}$ can be unrelated to collinear logarithms, and affect $\mathcal{B}_{s \gamma}$ by relative corrections in the range $[-0.3,1.9] \%$ with respect to the $\frac{m_{b}}{m_{s}}=50$ case, for $\mu_{b}=1.5 \mathrm{GeV}$ and $E_{0}=1.6 \mathrm{GeV}$. Numerically, we can reproduce this range by performing a replacement

$$
\ln \frac{m_{b}}{m_{s}} \rightarrow \kappa_{88} \ln 50 \quad \text { with } \quad \kappa_{88}=1.7 \pm 1.1
$$

in all the perturbative contributions proportional to $\left|C_{8}\left(\mu_{b}\right)\right|^{2}$.

In the following, we shall treat the quantities $\frac{\delta \Gamma_{c}}{\Gamma}(3.6), \kappa_{V}(3.9)$ and $\kappa_{88}$ (3.11) on equal footing with all the other parameters that $\mathcal{B}_{s \gamma}$ depends on. Since they account for all the non-perturbative effects estimated in refs. $[28,56]$, we shall no longer include the overall $\pm 5 \%$ non-perturbative uncertainty that entered the analysis of ref. [13] as an input from ref. [56]. This way we determine our updated SM predictions for $\mathcal{B}_{s \gamma}$ and $R_{\gamma}$ in the SM, namely

$$
\mathcal{B}_{s \gamma}=(3.40 \pm 0.17) \times 10^{-4} \quad \text { and } \quad R_{\gamma}=(3.35 \pm 0.16) \times 10^{-3},
$$

for $E_{0}=1.6 \mathrm{GeV}$. The overall uncertainties have been obtained by combining in quadrature the ones stemming from higher-order effects $( \pm 3 \%)$, interpolation in $m_{c}( \pm 3 \%)$, as well as the parametric uncertainty where all the non-perturbative ones are now contained. Not only $\frac{\delta \Gamma_{c}}{\Gamma}, \kappa_{V}$ and $\kappa_{88}$ but several other inputs parameterize non-perturbative effects, too, namely the collinear regulators (see above), as well as the HQET parameters that enter either directly or via the semileptonic phase-space factor $C$ (1.2). In the $\mathcal{B}_{s \gamma}$ case, our 
parametric uncertainty amounts to $\pm 2.5 \%$ at present. All the input parameters listed in appendix D of ref. [13] have been retained unchanged.

The overall uncertainty in $R_{\gamma}(3.12)$ amounts to $\pm 4.8 \%$, noticeably improved w.r.t. to $\pm 6.7 \%$ in ref. [12]. The main reason for the improvement comes from the updated estimate in ref. [28] of the non-perturbative uncertainty that stems from $\Lambda_{17}$ in eq. (3.9). Further improvement requires removing the $m_{c}$-interpolation, and re-considering the higher-order and parametric uncertainties. If they remain unchanged, the expected future accuracy in the SM prediction for $\mathcal{B}_{s \gamma}$ amounts to $\sqrt{3^{2}+2.5^{2}} \% \simeq 3.9 \%$, still somewhat behind the experimental expectation of $\pm 2.6 \%$ that was mentioned above eq. (1.1).

In many BSM theories, extra additive contributions $\Delta C_{7,8}$ to the Wilson coefficients of the operators $Q_{7}$ (1.6) and $Q_{8}$ (3.10) at the electroweak matching scale $\mu_{0}$ are the only relevant reason for shifting $\mathcal{B}_{s \gamma}$ and $R_{\gamma}$ away from the SM predictions. So long as no accidental cancellations occur, effects due to $\Delta C_{7,8}$ must be small whenever the current experimental constraints are satisfied. At such points in the BSM parameter spaces, $\mathcal{B}_{s \gamma}$ and $R_{\gamma}$ can accurately be calculated from the following simple linearized expressions

$$
\begin{aligned}
\mathcal{B}_{s \gamma} \times 10^{4} & =(3.40 \pm 0.17)-8.25 \Delta C_{7}-2.10 \Delta C_{8}, \\
R_{\gamma} \times 10^{3} & =(3.35 \pm 0.16)-8.08 \Delta C_{7}-2.06 \Delta C_{8},
\end{aligned}
$$

where $\mu_{0}=160 \mathrm{GeV}$ has been chosen. The above equations are updates of similar ones in eq. (10) of ref. [12]. Analytic formulae for the Wilson coefficients at $\mu_{0}$ in a wide class of BSM theories can be found in ref. [65].

In the specific case of the Two-Higgs-Doublet Model, eq. (3.13) can be replaced by expressions including all the NLO and NNLO QCD matching corrections [66]. The resulting 95\% C.L. lower bound from $R_{\gamma}$ on the charged Higgs boson mass in Model-II, evaluated along the same lines ${ }^{10}$ as in ref. [67], yields $800 \mathrm{GeV}$.

\section{Summary}

We reported on our calculation of the NNLO QCD corrections to $\mathcal{B}_{s \gamma}$ without interpolation in $m_{c}$, and presented final results for contributions originating from propagator diagrams with closed fermion loops on the gluon lines. They correspond either to the two-body $(s \gamma)$ or four-body $(s q \bar{q} \gamma)$ final states. In all the previously investigated cases, we confirmed the results from the literature, some of which had been obtained by a single group only. The new part comes from four diagrams with four-particle cuts that had not been determined before, as they are not included in the BLM approximation. Their contribution turns out to be tiny ( $\sim 0.1 \%$ of the decay rate) and quite well reproduced by our former interpolation algorithm.

In view of the recent progress in estimating the non-perturbative contributions, we performed an update of the phenomenological analysis within the SM. The obtained results yield $\mathcal{B}_{s \gamma}=(3.40 \pm 0.17) \times 10^{-4}$ and $R_{\gamma} \equiv \mathcal{B}_{(s+d) \gamma} / \mathcal{B}_{c \ell \bar{\nu}}=(3.35 \pm 0.16) \times 10^{-3}$

\footnotetext{
${ }^{10}$ The corresponding bound in the conclusions of ref. [67] amounted to $580 \mathrm{GeV}$.
} 
for $E_{0}=1.6 \mathrm{GeV}$. The main improvement in the uncertainty came from the analysis in ref. [28] where non-perturbative effects in the $Q_{1,2}-Q_{7}$ interference were re-analyzed.

The next contribution to suppressing the overall theoretical uncertainty is expected from the calculation of $\hat{G}_{17}^{(2)}$ and $\hat{G}_{27}^{(2)}$ for $E_{0}=0$ and at the physical value of $m_{c}$, thereby removing the need for $m_{c}$-interpolation in these quantities.

\section{Acknowledgments}

We are grateful to Alexander Smirnov and Johann Usovitsch for providing help to us as users of FIRE and KIRA, respectively. We would like to thank Gil Paz for extensive discussions concerning the non-perturbative contributions. The research of AR and MS has been supported by the Deutsche Forschungsgemeinschaft (DFG, German Research Foundation) under grant 396021762 - TRR 257 "Particle Physics Phenomenology after the Higgs Discovery". MM has been partially supported by the National Science Center, Poland, under the research project 2017/25/B/ST2/00191, and the HARMONIA project under contract UMO-2015/18/M/ST2/00518. This research was supported in part by the PL-Grid Infrastructure.

Note added in the proofs. While the present article was being reviewed for publication, a new paper [68] on non-perturbative effects in the $Q_{1,2^{-}} Q_{7}$ interference appeared on the arXiv. To replace the estimates of ref. [28] by those of ref. [68] in our approach, one would need to use $\kappa_{V}=1.7 \pm 0.8$ in eq. (3.9). This would shift our prediction for $\mathcal{B}_{\text {s }}$ from $(3.40 \pm 0.17) \times 10^{-4}$ to $(3.45 \pm 0.19) \times 10^{-4}$, and strengthen the constraint on $M_{H^{ \pm}}$even more. However, the extreme values of $\Lambda_{17}$ in ref. [68] originate from soft function models with quite a rich structure. Such soft functions are related to energy-momentum distributions of gluons inside the QCD ground states ( $B$ mesons), in which case encountering large numbers of extrema and zero points seems unlikely. Therefore, our preference is to retain $\kappa_{V}$ as it stands in eq. (3.9) for evaluating the SM predictions for $\mathcal{B}_{s \gamma}$ and $R_{\gamma}$.

\section{A Large- $z$ expansions and $\hat{G}_{47}^{(1)}$ with charm loops}

In this appendix, we present large- $z$ expansions of the renormalized contributions to $\hat{G}_{27}^{(2)}$ plotted in figures 3 and 4 . They are shown by the thin dashed lines reaching large values of $z$ in the corresponding plots. For the three plots in figure 3 that describe contributions from diagrams with closed loops of massless fermions, the respective expansions read

$$
\begin{aligned}
\Delta_{m=0}^{2-\mathrm{b}} \hat{G}_{27}^{(2)}= & 3\left[\frac{27650}{6561}+\frac{112}{243} L+\frac{8}{9} L^{2}+\frac{1}{z}\left(\frac{10427}{30375}-\frac{8}{135} \pi^{2}-\frac{572}{18225} L+\frac{38}{405} L^{2}\right)\right. \\
& \left.+\frac{1}{z^{2}}\left(\frac{19899293}{125023500}-\frac{8}{405} \pi^{2}-\frac{1628}{893025} L+\frac{86}{2835} L^{2}\right)\right]+\mathcal{O}\left(\frac{1}{z^{3}}\right), \\
\Delta_{m=0}^{4-\text { BLM }} \hat{G}_{27}^{(2)}= & 3\left[\frac{1}{z}\left(\frac{41}{108}-\frac{10}{243} \pi^{2}\right)+\frac{1}{z^{2}}\left(\frac{487}{3375}-\frac{2}{135} \pi^{2}\right)\right]+\mathcal{O}\left(\frac{1}{z^{3}}\right), \\
\Delta_{m=0}^{4-\text { B B-M }} \hat{G}_{27}^{(2)}= & 3\left[-\frac{32}{729}(1+L)+\frac{1}{z}\left(-\frac{941}{7290}+\frac{16}{1215} \pi^{2}\right)+\frac{1}{z^{2}}\left(-\frac{10852}{212625}+\frac{44}{8505} \pi^{2}\right)\right]+\mathcal{O}\left(\frac{1}{z^{3}}\right),
\end{aligned}
$$

where $L=\ln z$. The first expression above coincides with eq. (5.3) of ref. [22]. 
For the closed bottom loops (the left plot in figure 4), we find

$$
\begin{aligned}
\Delta_{m=m_{b}}^{2-\mathrm{b}} \hat{G}_{27}^{(2)} & =\frac{62210}{6561}+\frac{160}{729} \pi^{2}-\frac{16 \pi}{9 \sqrt{3}}-16 S_{2}+\left(\frac{464}{81}+\frac{160}{729} \pi^{2}-\frac{16 \pi}{9 \sqrt{3}}-16 S_{2}\right) L+\frac{8}{9} L^{2} \\
& +\frac{1}{z}\left(-\frac{30991}{10125}+\frac{656}{3645} \pi^{2}+\frac{4 \pi}{45 \sqrt{3}}+\frac{64}{405} \zeta_{3}+\frac{4}{5} S_{2}-\frac{32972}{18225} L+\frac{38}{405} L^{2}\right) \\
& +\frac{1}{z^{2}}\left(-\frac{38874763}{25004700}-\frac{8}{1701} \pi^{2}+\frac{26 \pi}{525 \sqrt{3}}+\frac{64}{2835} \zeta_{3}+\frac{12}{35} S_{2}-\frac{864896}{893025} L-\frac{418}{2835} L^{2}\right)+\mathcal{O}\left(\frac{1}{z^{3}}\right),
\end{aligned}
$$

where $S_{2}=\frac{4}{9 \sqrt{3}} \operatorname{Im}\left[\operatorname{Li}_{2}\left(e^{i \pi / 3}\right)\right]$. Finally, for the closed charm loops (the right plot in figure 4), the large- $z$ expansion reads

$$
\begin{aligned}
\Delta_{m=m_{c}}^{2-\mathrm{b}} \hat{G}_{27}^{(2)}= & \frac{11018}{6561}+\frac{128}{243} L+\frac{200}{243} L^{2}+\frac{1}{z}\left(\frac{5714}{54675}+\frac{7}{81} \zeta_{3}+\frac{2146}{18225} L+\frac{52}{405} L^{2}\right) \\
& +\frac{1}{z^{2}}\left(-\frac{62075113}{428652000}+\frac{469}{5184} \zeta_{3}-\frac{41987}{893025} L+\frac{92}{2835} L^{2}\right)+\mathcal{O}\left(\frac{1}{z^{3}}\right) .
\end{aligned}
$$

Our results in eqs. (A.2) and (A.3) agree with the numerical ones in eqs. (A.1) and (A.2) of ref. [26]. Analytical expressions for the leading terms agree with the findings of ref. [23].

Determining the renormalized results plotted in figures 3 and 4 required taking into account $\hat{G}_{47}^{(1) \text { bare }}$, i.e. three-loop counterterm diagrams with vertices proportional to $Q_{4}=\left(\bar{s}_{L} \gamma_{\mu} T^{a} b_{L}\right) \sum_{q}\left(\bar{q} \gamma^{\mu} T^{a} q\right)$. An expression for this quantity in eq. (2.4) of ref. [13] contains no contributions from closed loops of charm quarks, as all the other results in section 2 of that paper. Such contributions arise in the two-body channel only. They take the form

$$
\Delta_{m=m_{c}}^{2-\mathrm{b}} \hat{G}_{47}^{(1) \mathrm{bare}}=\frac{16}{81 \epsilon}-\frac{4}{243}+\frac{264 \pi^{2}-2186}{729} \epsilon+2 \operatorname{Re}[b(z)+\epsilon \tilde{b}(z)]+\mathcal{O}\left(\epsilon^{2}\right) .
$$

Small- $z$ expansion of the function $b(z)$ has been given in eq. (3.9) of ref. [69], while the large- $z$ expansion of $\operatorname{Re} b(z)$ can be found eq. (5.2) of ref. [22]. As far as $\tilde{b}(z)$ is concerned, we obtain the following expansions:

$$
\begin{aligned}
\operatorname{Re} \tilde{b}(z)= & \frac{1144}{729}-\frac{46}{243} \pi^{2}-\frac{8}{243} L-\frac{2}{81} L^{2}+\frac{1}{z}\left(\frac{10957}{60750}+\frac{212}{2025} L+\frac{1}{15} L^{2}\right) \\
& +\frac{1}{z^{2}}\left(\frac{491839}{41674500}+\frac{134}{33075} L+\frac{2}{63} L^{2}\right)+\mathcal{O}\left(\frac{1}{z^{3}}\right), \\
\operatorname{Re} \tilde{b}(z)= & \left(\frac{44}{3}-\frac{16}{9} \pi^{2}-\frac{40}{9} \zeta_{3}+\frac{16}{9} L-\frac{8}{9} L^{2}\right) z+\left(\frac{304}{81}-\frac{128}{27} \ln 2-\frac{32}{27} L\right) \pi^{2} z^{\frac{3}{2}} \\
& +\left(\frac{53}{3}-\frac{20}{27} \pi^{2}+\frac{14}{3} L-\frac{32}{27} \pi^{2} L+\frac{10}{9} L^{2}-\frac{4}{9} L^{3}\right) z^{2}-\frac{80}{27} \pi^{2} z^{\frac{5}{2}} \\
& +\left(\frac{6830}{729}-\frac{292}{243} \pi^{2}+\frac{80}{27} \zeta_{3}+\frac{68}{243} L+\frac{64}{81} \pi^{2} L-\frac{124}{27} L^{2}+\frac{16}{9} L^{3}\right) z^{3}+\frac{88}{135} \pi^{2} z^{\frac{7}{2}} \\
& +\left(\frac{1944727}{121500}-\frac{304}{405} \pi^{2}+\frac{32}{9} \zeta_{3}-\frac{17239}{2025} L-\frac{80}{27} L^{2}+\frac{16}{9} L^{3}\right) z^{4}+\frac{272}{2835} \pi^{2} z^{\frac{9}{2}} \\
& +\left(\frac{34017647}{833490}-\frac{1018}{189} \pi^{2}+\frac{80}{9} \zeta_{3}-\frac{113308}{3969} L-\frac{182}{27} L^{2}+\frac{40}{9} L^{3}\right) z^{5}+\mathcal{O}\left(z^{\frac{11}{2}}\right) .
\end{aligned}
$$

No explicit expressions for the expansions of $\tilde{b}(z)$ have so far been published, even though this function must have been used for UV renormalization in ref. [26]. 
Open Access. This article is distributed under the terms of the Creative Commons Attribution License (CC-BY 4.0), which permits any use, distribution and reproduction in any medium, provided the original author(s) and source are credited.

\section{References}

[1] S.L. Glashow, J. Iliopoulos and L. Maiani, Weak Interactions with Lepton-Hadron Symmetry, Phys. Rev. D 2 (1970) 1285 [InSPIRE].

[2] J. Aebischer, W. Altmannshofer, D. Guadagnoli, M. Reboud, P. Stangl and D.M. Straub, B-decay discrepancies after Moriond 2019, Eur. Phys. J. C 80 (2020) 252 [arXiv: 1903.10434] [INSPIRE].

[3] CLEO collaboration, Branching fraction and photon energy spectrum for $b \rightarrow s \gamma$, Phys. Rev. Lett. 87 (2001) 251807 [hep-ex/0108032] [INSPIRE].

[4] BABAR collaboration, Measurement of the $B \rightarrow X_{s} \gamma$ branching fraction and photon energy spectrum using the recoil method, Phys. Rev. D 77 (2008) 051103 [arXiv:0711.4889] [INSPIRE].

[5] BABAR collaboration, Exclusive Measurements of $b \rightarrow s \gamma$ Transition Rate and Photon Energy Spectrum, Phys. Rev. D 86 (2012) 052012 [arXiv:1207.2520] [InSPIRE].

[6] BaBAR collaboration, Precision Measurement of the $B \rightarrow X_{s} \gamma$ Photon Energy Spectrum, Branching Fraction and Direct CP Asymmetry $A_{C P}\left(B \rightarrow X_{s+d} \gamma\right)$, Phys. Rev. Lett. 109 (2012) 191801 [arXiv:1207.2690] [INSPIRE].

[7] BeLLe collaboration, Measurement of the $\bar{B} \rightarrow X_{s} \gamma$ Branching Fraction with a Sum of Exclusive Decays, Phys. Rev. D 91 (2015) 052004 [arXiv:1411.7198] [INSPIRE].

[8] BelLe collaboration, Measurement of the inclusive $B \rightarrow X_{s+d} \gamma$ branching fraction, photon energy spectrum and HQE parameters, in 38th International Conference on High Energy Physics, (2016) [arXiv: 1608.02344] [INSPIRE].

[9] HFLAV collaboration, Averages of b-hadron, c-hadron and $\tau$-lepton properties as of 2018, arXiv:1909.12524 [INSPIRE].

[10] BeLle-II collaboration, The Belle II Physics Book, PTEP 2019 (2019) 123 C01 [Erratum ibid. 2020 (2020) 029201] [arXiv: 1808.10567] [INSPIRE].

[11] A. Ishikawa, Prospects for future Belle-II measurements, talk at the 7th Workshop on Rare Semileptonic B Decays, Lyon, France, 4-6 September 2019 [https://indico.in2p3.fr/event/18646].

[12] M. Misiak et al., Updated NNLO QCD predictions for the weak radiative B-meson decays, Phys. Rev. Lett. 114 (2015) 221801 [arXiv:1503.01789] [INSPIRE].

[13] M. Czakon, P. Fiedler, T. Huber, M. Misiak, T. Schutzmeier and M. Steinhauser, The $\left(Q_{7}, Q_{1,2}\right)$ contribution to $\bar{B} \rightarrow X_{s} \gamma$ at $\mathcal{O}\left(\alpha_{\mathrm{s}}^{2}\right)$, JHEP 04 (2015) 168 [arXiv: 1503.01791] [INSPIRE].

[14] A. Alberti, P. Gambino, K.J. Healey and S. Nandi, Precision Determination of the Cabibbo-Kobayashi-Maskawa Element $V_{c b}$, Phys. Rev. Lett. 114 (2015) 061802 [arXiv: 1411.6560] [INSPIRE].

[15] M. Misiak and M. Steinhauser, Three loop matching of the dipole operators for $b \rightarrow s \gamma$ and $b \rightarrow s g$, Nucl. Phys. B 683 (2004) 277 [hep-ph/0401041] [InSPIRE]. 
[16] M. Czakon, U. Haisch and M. Misiak, Four-Loop Anomalous Dimensions for Radiative Flavour-Changing Decays, JHEP 03 (2007) 008 [hep-ph/0612329] [INSPIRE].

[17] I.R. Blokland, A. Czarnecki, M. Misiak, M. Ślusarczyk and F. Tkachov, The Electromagnetic dipole operator effect on $\bar{B} \rightarrow X_{s} \gamma$ at $O\left(\alpha_{s}^{2}\right)$, Phys. Rev. D 72 (2005) 033014 [hep-ph/0506055] [INSPIRE].

[18] K. Melnikov and A. Mitov, The photon energy spectrum in $\bar{B} \rightarrow X_{s}+\gamma$ in perturbative $Q C D$ through $O\left(\alpha_{s}^{2}\right)$, Phys. Lett. B 620 (2005) 69 [hep-ph/0505097] [INSPIRE].

[19] H.M. Asatrian, A. Hovhannisyan, V. Poghosyan, T. Ewerth, C. Greub and T. Hurth, NNLL $Q C D$ contribution of the electromagnetic dipole operator to $\Gamma\left(\bar{B} \rightarrow X_{s} \gamma\right)$, Nucl. Phys. B $\mathbf{7 4 9}$ (2006) 325 [hep-ph/0605009] [INSPIRE].

[20] H.M. Asatrian, T. Ewerth, A. Ferroglia, P. Gambino and C. Greub, Magnetic dipole operator contributions to the photon energy spectrum in $\bar{B} \rightarrow X_{s} \gamma$ at $O\left(\alpha_{s}^{2}\right)$, Nucl. Phys. B 762 (2007) 212 [hep-ph/0607316] [inSPIRE].

[21] H.M. Asatrian, T. Ewerth, H. Gabrielyan and C. Greub, Charm quark mass dependence of the electromagnetic dipole operator contribution to $\bar{B} \rightarrow X_{s} \gamma$ at $O\left(\alpha_{s}^{2}\right)$, Phys. Lett. B 647 (2007) 173 [hep-ph/0611123] [INSPIRE].

[22] M. Misiak and M. Steinhauser, NNLO QCD corrections to the $\bar{B} \rightarrow X_{s} \gamma$ matrix elements using interpolation in $m_{c}$, Nucl. Phys. B 764 (2007) 62 [hep-ph/0609241] [INSPIRE].

[23] M. Misiak and M. Steinhauser, Large- $m_{c}$ Asymptotic Behaviour of $O\left(\alpha_{s}^{2}\right)$ Corrections to $\bar{B} \rightarrow X_{s} \gamma$, Nucl. Phys. B 840 (2010) 271 [arXiv:1005.1173] [InSPIRE].

[24] Z. Ligeti, M.E. Luke, A.V. Manohar and M.B. Wise, The $\bar{B} \rightarrow X_{s} \gamma$ photon spectrum, Phys. Rev. D 60 (1999) 034019 [hep-ph/9903305] [INSPIRE].

[25] K. Bieri, C. Greub and M. Steinhauser, Fermionic NNLL corrections to $b \rightarrow s \gamma$, Phys. Rev. D 67 (2003) 114019 [hep-ph/0302051] [INSPIRE].

[26] R. Boughezal, M. Czakon and T. Schutzmeier, NNLO fermionic corrections to the charm quark mass dependent matrix elements in $\bar{B} \rightarrow X_{s} \gamma$, JHEP 09 (2007) 072 [arXiv: 0707.3090] [INSPIRE].

[27] M. Misiak and M. Poradziński, Completing the Calculation of BLM corrections to $\bar{B} \rightarrow X_{s} \gamma$, Phys. Rev. D 83 (2011) 014024 [arXiv: 1009.5685] [InSPIRE].

[28] A. Gunawardana and G. Paz, Reevaluating uncertainties in $\bar{B} \rightarrow X_{s} \gamma$ decay, JHEP 11 (2019) 141 [arXiv: 1908. 02812] [INSPIRE].

[29] P. Nogueira, Automatic Feynman graph generation, J. Comput. Phys. 105 (1993) 279 [INSPIRE].

[30] J. Kublbeck, M. Böhm and A. Denner, Feyn Arts: Computer Algebraic Generation of Feynman Graphs and Amplitudes, Comput. Phys. Commun. 60 (1990) 165 [INSPIRE].

[31] T. Hahn, Generating Feynman diagrams and amplitudes with FeynArts 3, Comput. Phys. Commun. 140 (2001) 418 [hep-ph/0012260] [INSPIRE].

[32] B. Ruijl, T. Ueda and J. Vermaseren, FORM version 4.2, arXiv:1707.06453 [InSPIRE].

[33] F.V. Tkachov, A Theorem on Analytical Calculability of Four Loop Renormalization Group Functions, Phys. Lett. B 100 (1981) 65 [inSPIRE].

[34] K.G. Chetyrkin and F.V. Tkachov, Integration by Parts: The Algorithm to Calculate $\beta$-functions in 4 Loops, Nucl. Phys. B 192 (1981) 159 [INSPIRE]. 
[35] S. Laporta, High precision calculation of multiloop Feynman integrals by difference equations, Int. J. Mod. Phys. A 15 (2000) 5087 [hep-ph/0102033] [InSPIRE].

[36] P. Maierhöfer, J. Usovitsch and P. Uwer, Kira - A Feynman integral reduction program, Comput. Phys. Commun. 230 (2018) 99 [arXiv:1705.05610] [InSPIRE].

[37] P. Maierhöfer and J. Usovitsch, Kira 1.2 Release Notes, arXiv:1812.01491 [InSPIRE].

[38] A.V. Smirnov, FIRE5: a C++ implementation of Feynman Integral REduction, Comput. Phys. Commun. 189 (2015) 182 [arXiv:1408.2372] [INSPIRE].

[39] A.V. Smirnov and F.S. Chuharev, FIRE6: Feynman Integral REduction with Modular Arithmetic, arXiv:1901.07808 [INSPIRE].

[40] R.N. Lee, Presenting LiteRed: a tool for the Loop InTEgrals REDuction, arXiv:1212.2685 [INSPIRE].

[41] R.N. Lee, LiteRed 1.4: a powerful tool for reduction of multiloop integrals, J. Phys. Conf. Ser. 523 (2014) 012059 [arXiv: 1310.1145] [INSPIRE].

[42] A.V. Kotikov, Differential equations method: New technique for massive Feynman diagrams calculation, Phys. Lett. B 254 (1991) 158 [INSPIRE].

[43] E. Remiddi, Differential equations for Feynman graph amplitudes, Nuovo Cim. A 110 (1997) 1435 [hep-th/9711188] [INSPIRE].

[44] T. Gehrmann and E. Remiddi, Differential equations for two loop four point functions, Nucl. Phys. B $\mathbf{5 8 0}$ (2000) 485 [hep-ph/9912329] [InSPIRE].

[45] M. Misiak, A. Rehman and M. Steinhauser, NNLO QCD counterterm contributions to $\bar{B} \rightarrow X_{s \gamma}$ for the physical value of $m_{c}$, Phys. Lett. B $\mathbf{7 7 0}$ (2017) 431 [arXiv:1702.07674] [INSPIRE].

[46] A. Rehman, Strong Interaction Corrections to the Weak Radiative B-Meson Decay at Order $O\left(\alpha_{s}^{2}\right)$ with Exact Dependence on the c-Quark Mass, Ph.D. Thesis, University of Warsaw (2015) [http://depotuw.ceon.pl/handle/item/1197].

[47] V.A. Smirnov, Applied asymptotic expansions in momenta and masses, Springer Tracts Mod. Phys. 177 (2002) 1.

[48] R. Harlander, T. Seidensticker and M. Steinhauser, Complete corrections of $O\left(\alpha \alpha_{s}\right)$ to the decay of the $Z$ boson into bottom quarks, Phys. Lett. B 426 (1998) 125 [hep-ph/9712228] [INSPIRE].

[49] T. Seidensticker, Automatic application of successive asymptotic expansions of Feynman diagrams, in 6th International Workshop on New Computing Techniques in Physics Research: Software Engineering, Artificial Intelligence Neural Nets, Genetic Algorithms, Symbolic Algebra, Automatic Calculation, (1999) [hep-ph/9905298] [INSPIRE].

[50] M. Czaja, T. Huber, G. Mishima, M. Misiak, A. Rehman and M. Steinhauser, in preparation.

[51] P.N. Brown, G.D. Byrne and A.C. Hindmarsh, zvoDE, http://netlib.sandia.gov/ode/zvode.f.

[52] Y. Hida, X.S. Li and D.H. Bailey, QD computation package, http://crd-legacy.lbl.gov/ dhbailey/mpdist.

[53] S.J. Brodsky, G. Lepage and P.B. Mackenzie, On the Elimination of Scale Ambiguities in Perturbative Quantum Chromodynamics, Phys. Rev. D 28 (1983) 228 [INSPIRE]. 
[54] Belle collaboration, Measurements of isospin asymmetry and difference of direct CP asymmetries in inclusive $B \rightarrow X_{s} \gamma$ decays, Phys. Rev. D 99 (2019) 032012 [arXiv: 1807.04236] [INSPIRE].

[55] S.J. Lee, M. Neubert and G. Paz, Enhanced Non-local Power Corrections to the $\bar{B} \rightarrow X_{s} \gamma$ Decay Rate, Phys. Rev. D 75 (2007) 114005 [hep-ph/0609224] [INSPIRE].

[56] M. Benzke, S.J. Lee, M. Neubert and G. Paz, Factorization at Subleading Power and Irreducible Uncertainties in $\bar{B} \rightarrow X_{s} \gamma$ Decay, JHEP 08 (2010) 099 [arXiv:1003.5012] [INSPIRE].

[57] M. Misiak, $\bar{B} \rightarrow X_{s} \gamma$ : Current Status, Acta Phys. Polon. B 40 (2009) 2987 [arXiv: 0911.1651] [INSPIRE].

[58] L.A. Goodman, On the Exact Variance of Products, J. Am. Statist. Assoc. 55 (1960) 708.

[59] Particle Data Group collaboration, Review of Particle Physics, Phys. Rev. D 98 (2018) 030001 [INSPIRE].

[60] http://pdg.lbl.gov/2019/listings/rpp2019-list-B-plus-minus-B0-admixture.pdf, update on p. 66 .

[61] M.B. Voloshin, Large $\mathcal{O}\left(m_{c}^{-2}\right)$ nonperturbative correction to the inclusive rate of the decay $B \rightarrow X_{s} \gamma$, Phys. Lett. B 397 (1997) 275 [hep-ph/9612483] [INSPIRE].

[62] G. Buchalla, G. Isidori and S.J. Rey, Corrections of order $\Lambda_{Q C D}^{2} / m_{c}^{2}$ to inclusive rare $B$ decays, Nucl. Phys. B 511 (1998) 594 [hep-ph/9705253] [INSPIRE].

[63] A. Kapustin, Z. Ligeti and H. Politzer, Leading logarithms of the b quark mass in inclusive $B \rightarrow X_{s} \gamma$ decay, Phys. Lett. B 357 (1995) 653 [hep-ph/9507248] [InSPIRE].

[64] A. Ferroglia and U. Haisch, Chromomagnetic Dipole-Operator Corrections in $\bar{B} \rightarrow X_{s} \gamma$ at $O\left(\beta_{0} \alpha_{s}^{2}\right)$, Phys. Rev. D 82 (2010) 094012 [arXiv: 1009.2144] [INSPIRE].

[65] C. Bobeth, M. Misiak and J. Urban, Matching conditions for $b \rightarrow s \gamma$ and $b \rightarrow s$ gluon in extensions of the standard model, Nucl. Phys. B 567 (2000) 153 [hep-ph/9904413] [INSPIRE].

[66] T. Hermann, M. Misiak and M. Steinhauser, $\bar{B} \rightarrow X_{s} \gamma$ in the Two Higgs Doublet Model up to Next-to-Next-to-Leading Order in QCD, JHEP 11 (2012) 036 [arXiv:1208.2788] [INSPIRE].

[67] M. Misiak and M. Steinhauser, Weak radiative decays of the $B$ meson and bounds on $M_{H^{ \pm}}$in the Two-Higgs-Doublet Model, Eur. Phys. J. C 77 (2017) 201 [arXiv:1702. 04571] [InSPIRE].

[68] M. Benzke and T. Hurth, Resolved $1 / m_{b}$ contributions to $b \rightarrow$ sll and $b \rightarrow s \gamma$, arXiv:2006.00624 [INSPIRE].

[69] A.J. Buras, A. Czarnecki, M. Misiak and J. Urban, Completing the NLO QCD calculation of $\bar{B} \rightarrow X_{s} \gamma$, Nucl. Phys. B 631 (2002) 219 [hep-ph/0203135] [INSPIRE]. 